\title{
Speaking up for Mental Health
}

\section{Three advocates for combatting mental-health issues in academia share ideas for making a more supportive community.}

\author{
By Zoë Ayres, Andrea Welsh, and Joeri Tijdink
}

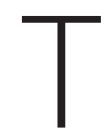
hose struggling with mental health issues have always been stigmatized, and academics are no exception. But researchers are actively pushing for change, and sessions devoted to mental health are becoming more common at scientific conferences.

As the coronavirus pandemic rages on, many communities around the world are seeing a sharp increase in the prevalence of anxiety, depression, and other mental illnesses. This problem could exacerbate the already high prevalence of depression among Ph.D. students. To bring attention to the issue, we asked three advocates for mental health awareness to share their perspectives. Zoë Ayres, an industrial research scientist in the UK, explains why candid conversations are needed to break the stigma surrounding mental health struggles; Andrea Welsh, a postdoctoral researcher at the University of Pittsburgh, advocates for support communities in physics; and Joeri Tijdink, a psychiatrist and mental health researcher in the Netherlands discusses the burden that publication metrics place on academics. - Katherine Wright

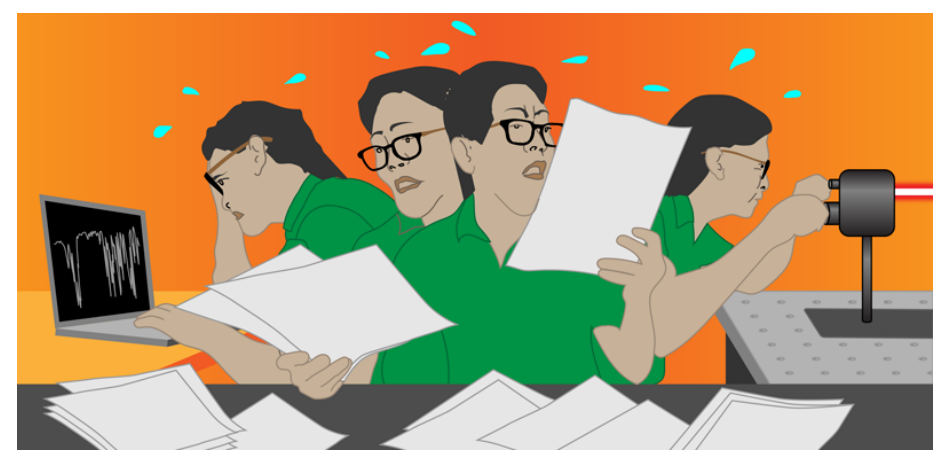

The pressure to constantly churn out new papers is a leading cause of mental health issues among academics.

Credit: APS/Alan Stonebraker

\section{Why academics need to talk about mental health} Mental health concerns among academics had reached epidemic proportions way before COVID-19 came along, with approximately $50 \%$ of Ph.D. students across all fields experiencing anxiety and depression during their studies. It is no wonder the situation has been referred to as a crisis. And the battle with mental health does not disappear on completion of a Ph.D. With the pressure to publish, the precarity of contracts, the existence of systemic sexism and racism, and the frequent rejection of grant applications, combined with the pervasive-incorrect-notion that being passionate about research means working every hour of every day, it is clear why many academics struggle.

In order to cure this crisis, we must talk openly about our mental health. Starting such a conversation has been the motivation behind much of my mental health advocacy work, which includes creating mental health posters and running the 100 voices campaign on Twitter. The campaign features the mental health journeys of 100 researchers, with the aim of breaking the stigma around mental health. It is through efforts like this one, which involve actively talking about mental health, that we can start to identify the themes and factors that cause and sustain mental health problems in our fields.

This conversation cannot be one-sided-senior management at our universities and institutions must listen to those suffering with mental health problems and work with them, and vice versa, to find solutions. All too often, the onus is placed on the individual to "be more resilient" or to "seek help" themselves, but there are many ways that universities can support both staff and students, as highlighted, for example, in one of my posters. By engaging, not blaming, we can open the door for institutional and cultural change. 


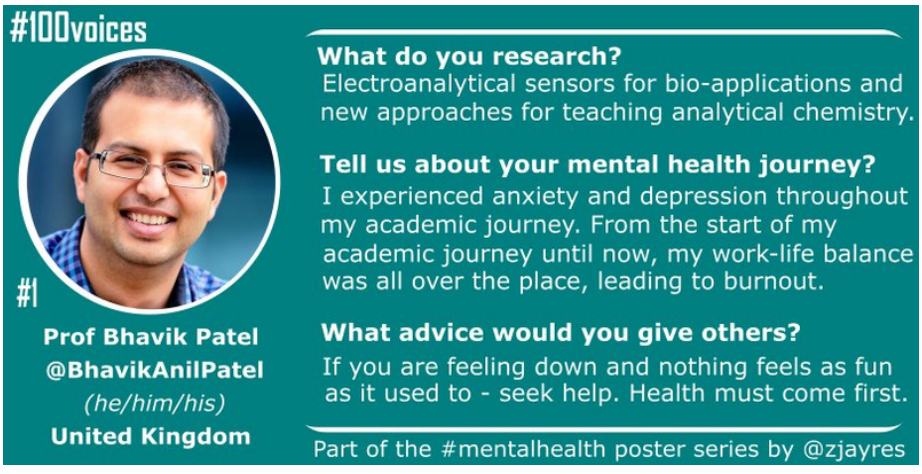

The \#100voices campaign on Twitter by Zoë Ayres aimed to kickstart a conversation about mental health. The campaign, which ended in July, featured posters about the mental health issues faced by researchers from around the world.

Credit: Twitter/@ZJAYRES

While making sure our peers are happy is simply the right thing to do, there are other important reasons to talk about mental health. For example, poor mental health can lead to burnout-a physical or mental collapse caused by overwork or stress-which can impede productivity and project progress. Unsurprisingly, those that feel alone or unsupported often end up going to industry or to other careers entirely. Sadly, loss of talent also happens by suicide. Clearly something needs to change.

Talking about mental health is particularly important for postgraduates, who miss out on mental health support, as the focus tends to be on undergraduate mental health provision. So often these students see their professors as highly successful academics, with no visible issues or concerns; they cannot see the crack-filled facade many of us have built around ourselves. This problem can amplify feelings of impostor syndrome, which leads to feelings of inadequacy, another factor that causes researchers to change careers. By keeping our struggles quiet, we reinforce the notion that those who are different don't belong.

Of course, speaking out is hard. Many stay quiet because of concerns over future employability or discrimination. But those of us that do feel able to talk about our experiences must make our voices heard. It is in small, brave steps-like taking part in a campaign-that we can start to make a difference and encourage others to do the same. In doing so-hopefully-we can break the cycle before poor mental health, like COVID-19, reaches pandemic scales. - Zoë Ayres

\section{Supportive Groups Are Vital for Mental Health}

When it comes to combating mental health problems, individual talk therapy is the option many people turn to. While this treatment can be effective, it doesn't remove the underlying triggers for mental health problems, such as a toxic work culture or the presence of system inequalities against those with marginalized identities. Removing those triggers is crucial to reducing the high prevalence of poor mental health of academics. To do that, we need supportive work environments where we can share our experiences. We also need conscientious and responsive leaders that actively address problems as they arise.

When I was an undergraduate in the late 2000s, mental health was rarely discussed and then only in hushed whispers. It wasn't until a physicist friend of mine happened to mention to me that he was seeing a university counselor that I even knew those services existed.

At the time, I was suffering from complex PTSD and depression, and I was too nervous to seek counselling. But the information stayed with me, and when I reached a low point in my life during graduate school, I knew where to turn. Seeking therapy is one my best decisions; it helped me survive and complete my Ph.D. But without the advice from my friend-someone with whom I was comfortable discussing my mental health-I would have been lost.

For undergraduates, support networks can take the form of a classroom of peers, a university organization, or fellow dormers. For graduates, life can be much more isolating. Interactions with others predominantly occur in work environments, such as an office or a lab, where it may feel inappropriate to discuss personal matters. As a result, it can be hard, if not impossible, to find others experiencing similar feelings without broadcasting your own pain publicly-a terrifying prospect for many.

But in 2017, I did exactly that when I spoke about my depression at a session I co-organized for that year's APS March Meeting on mental health and graduate school. Since then, many students and early career researchers have approached me with their 
own stories. These conversations spurred me to create a "Mental Health in Physics" Google Group, with an associated Slack channel, that currently connects over 100 physicists. In the group, people talk about their mental health issues, ask for advice, share resources, and-importantly-support each other.

It's nice to be able to laugh with someone about the side effects of forgetting to take pills, without worrying about being judged and without being offered unsolicited advice, the prospect of which can make it hard to open up. The group also discusses how to deal with stressful work situations and how the physics community can do better at combating mental health problems. The discussions focus on how to avoid toxic or unresponsive supervisors and how to deal with issues such as long work hours.

As this group continues to grow, I hope that these conversations will lead to lasting systematic changes in physics. We need proper management training for Principal Investigators so that their group members flourish rather than wither away. We need support from physics departments when we are taken advantage of or forgotten. And now, more than ever, when isolation and racial injustice are at the forefront of our collective consciousness, we need support communities: places to remember we are not alone. - Andrea Welsh

\section{Removing the Mental Burden of Publication Metrics}

In academia, the mental health statistics are startling. As Ayres mentioned, studies carried out in China and Belgium found that roughly half of Ph.D. students display signs of depression and anxiety, while a survey of Ph.D. students from around the world found that $36 \%$ of them have sought clinical support for mental health symptoms. Furthermore, in the Netherlands, in a study I was involved in, we found that almost one quarter of biomedical professors showed signs of burnout, such as emotional exhaustion. And another study found the same burnout signs for one third of Ph.D. students.

So, what is behind this mental health problem? Growing evidence suggests that the answer is publication numbers and publication output. Academia revolves around numbers. For example, academic prestige and advancement depend on the number of papers a researcher publishes, the number of times those papers are cited, and the impact factor of the journals the papers appear in. Academics with higher publication numbers

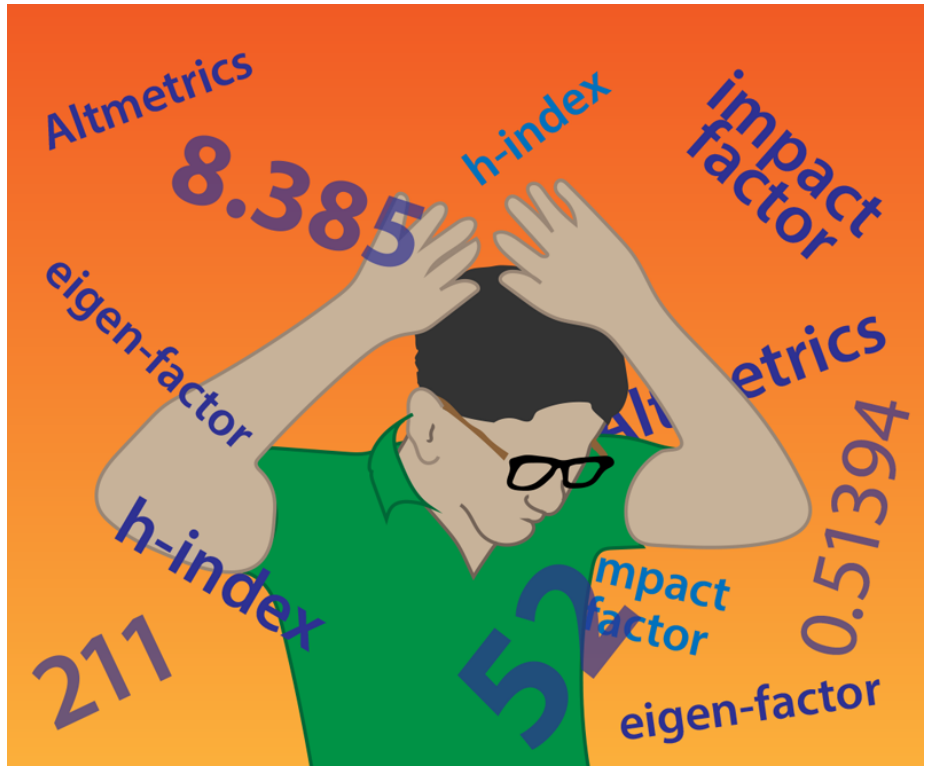

For many researchers, advancement depends on numbers, such as the number of papers they publish and the number of times those papers are cited. The pressure many feel to increase these numbers can lead to poor mental health and burnout.

Credit: APS/Alan Stonebraker

are perceived as being more successful researchers. And the pressure that those metrics put on researchers to churn out papers, for example, can lead to burnout. This so-called publication pressure is highest among postdocs and assistant professors, who believe they must "publish or perish." These untenured academics are the most burdened by the current number-based assessment. Consequently, they are the most likely to experience a deteriorating mental health.

The unidimensional focus on publications has other consequences. For example, it can discourage participation in other-equally important-academic tasks, such as supervising students, teaching undergraduates, and speaking publicly about the societal relevance of their work. These activities are often unaccounted for in performance metrics, and the time and energy put into these activities is rarely rewarded.

Despite these problems, there is room for optimism. Over the last 10 years, several initiatives have looked at alternative forms of academic advancement. They include the Declaration on Research Assessment (DORA), which recognizes the need to 
improve the evaluation of scholarly research outputs, and the Hong Kong manifesto, a 2019 report that emphasizes the need to value academic duties, such as mentoring, conducting outreach activities, reporting results transparently, and engaging in open science practices. One of the recommendations to come out of the initiatives is to account for all academic duties-not just publishing papers and gaining funding-in performance reviews.

These initiatives highlight the importance of changing how we assess academic performance, and they have sparked discussions on how academic performance is judged. These conversations are motivating changes in institutional practices that should lead to fair and constructive academic assessment criteria.

I am convinced that these changes will improve mental health among academics, specifically early career researchers. First, these measures should lessen the pressure to publish.

Researches will have more time to carry out their studies, allowing them to incorporate responsible research practices, such as open science practices. Furthermore, they will reduce the push to publish fast, which can (unintentionally) result in corner cutting. Not having to constantly churn out papers would lessen stress and curtail competition, creating a healthier research culture. As a result, science might slow down, reducing the pressure on academics to quickly produce new results. That could lead to more rigorous methods that may improve the validity of research results. A slow-down could also give academics time to have more fun in their work. Such a change would certainly lead to happier, healthier academics and a more constructive and positive research environment. - Joeri Tijdink

Zoë Ayres: Birmingham, United Kingdom

Andrea Welsh: University of Pittsburgh, Pittsburgh, PA, US

Joeri Tijdink: VU Amsterdam and University Medical Center Amsterdam, Netherlands 